\title{
ATLAS Commissioning and Physics with Early Data
}

\author{
Paul Bell \\ The University of Manchester \\ on behalf of the ATLAS collaboration \\ Lake Louise Winter Institute \\ February 2008
}

1. ATLAS status and commissioning pre-LHC startup

2. Commissioning and physics with early data 


\section{1: Status and Commissioning pre-LHC Startup}




\section{A word on the LHC}

- Machine closure end of May; beam commissioning June 2008

- Currently in cooling down phase: cooling each sector to $1.9 \mathrm{~K}$ takes $\sim 8$ weeks

- Four commissioning phases

- A: - establish first circulating beam $\rightarrow$ first $7 \mathrm{TeV}$ collisions ( 2 months)

- pilot physics run (1 month) with luminosity $\sim 10^{31} \mathrm{~cm}^{-2} \mathrm{~s}^{-1}$

- should deliver $\sim 3 \mathrm{pb}^{-1}$ integrated luminosity

- B: move to $75 \mathrm{~ns}$ bunch spacing, up to 936 bunches/beam $\left(\sim 10^{32} \mathrm{~cm}^{-2} \mathrm{~s}^{-1}\right)$

- C: move to $25 \mathrm{~ns}$ bunch spacing $\left(\sim 10^{33} \mathrm{~cm}^{-2} \mathrm{~s}^{-1}\right)$ $<$ shutdown>

$\rightarrow$ towards full current $\left(10^{34} \mathrm{~cm}^{-2} \mathrm{~s}^{-1}\right)$

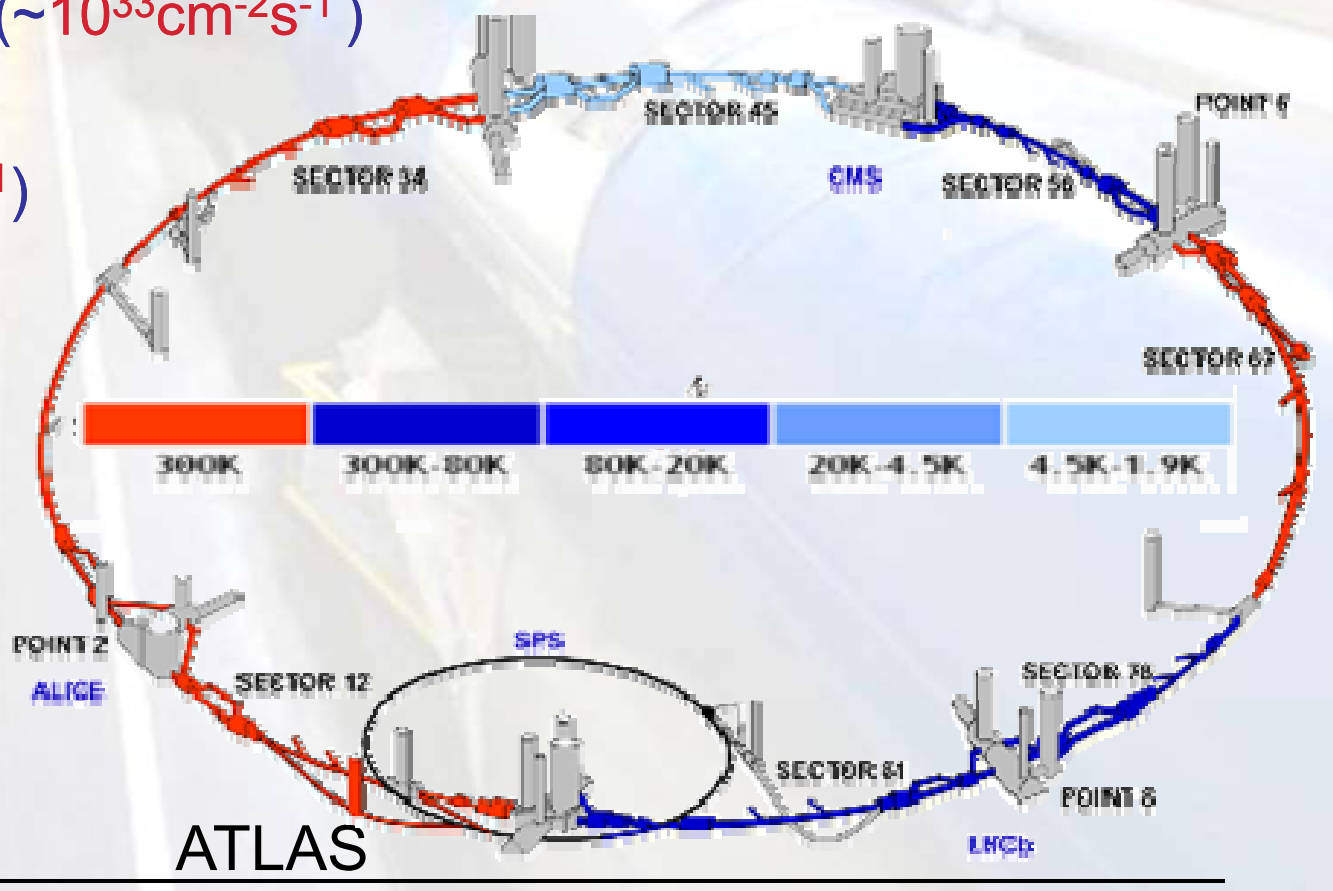




\section{ATLAS Basics}

$25 \mathrm{~m}$ diameter, $46 \mathrm{~m}$ long, weighing $7000 \mathrm{~T}$, with $3000 \mathrm{~km}$ of cables and $10^{8}$ channels

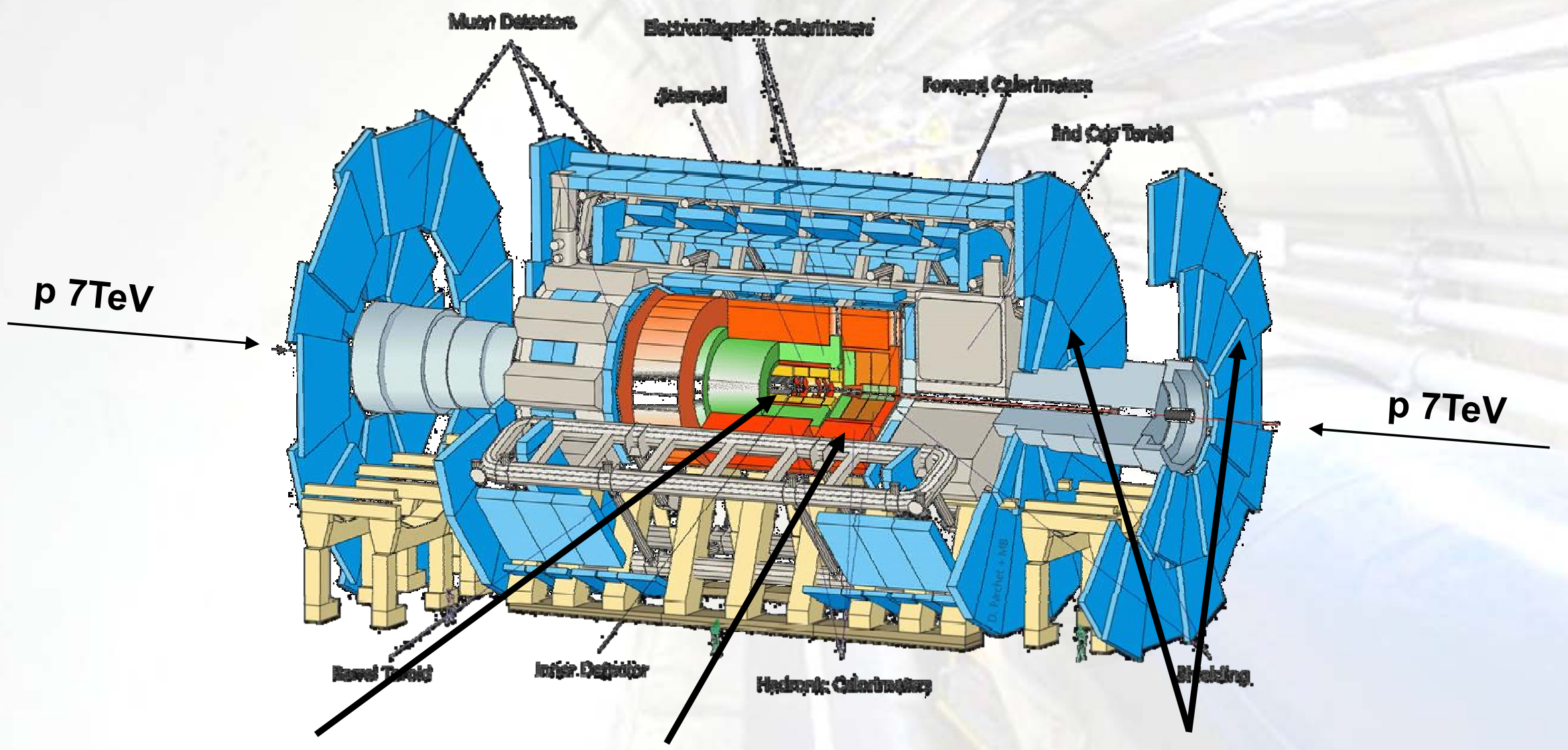

Tracking $(|\eta|<2.5, B=2 T)$ :

- Silicon pixels and strips

- Transition Radiation Tracker
Calorimetry $(|\eta|<5)$

$\mathrm{EM}: \mathrm{Pb}-\mathrm{LAr}$

HAD: Fe/scintillator

(central), Cu/W-LAr (fwd)
Muon spectrometer $(|\eta|<2.7, B=4 \mathrm{~T}$ max) Toroid magnet system with precision and trigger chambers 


\section{ATLAS Commissioning Activities}

\section{"Online"}

1. Commissioning of individual subsystems in pit (almost finished)

2. Integration of subsystems into ATLAS trigger and DAQ system (on-going)

Several global commissioning runs using cosmic rays in 2007:

- operate the whole experiment

- achieve stable running for long periods

- exercise Trigger and DAQ (data flow, run control, configuration)

- operate control room as if data taking

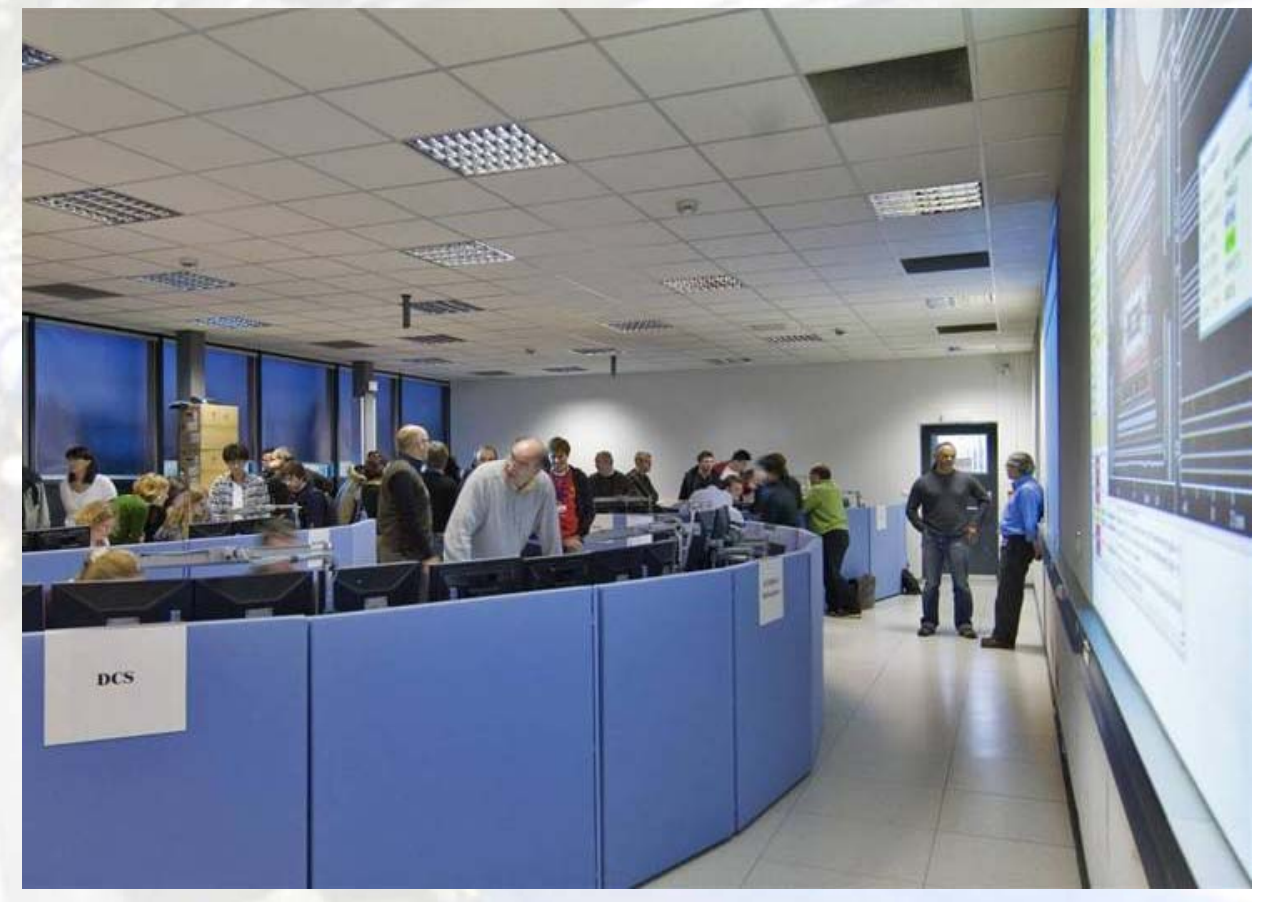

\section{Should not forget "Offline"}

- Full Dress Rehearsal (FDR):

a "stress test" of the full data processing and analysis chain from point-1 to the end user 


\section{Global Commissioning Runs with Cosmics}

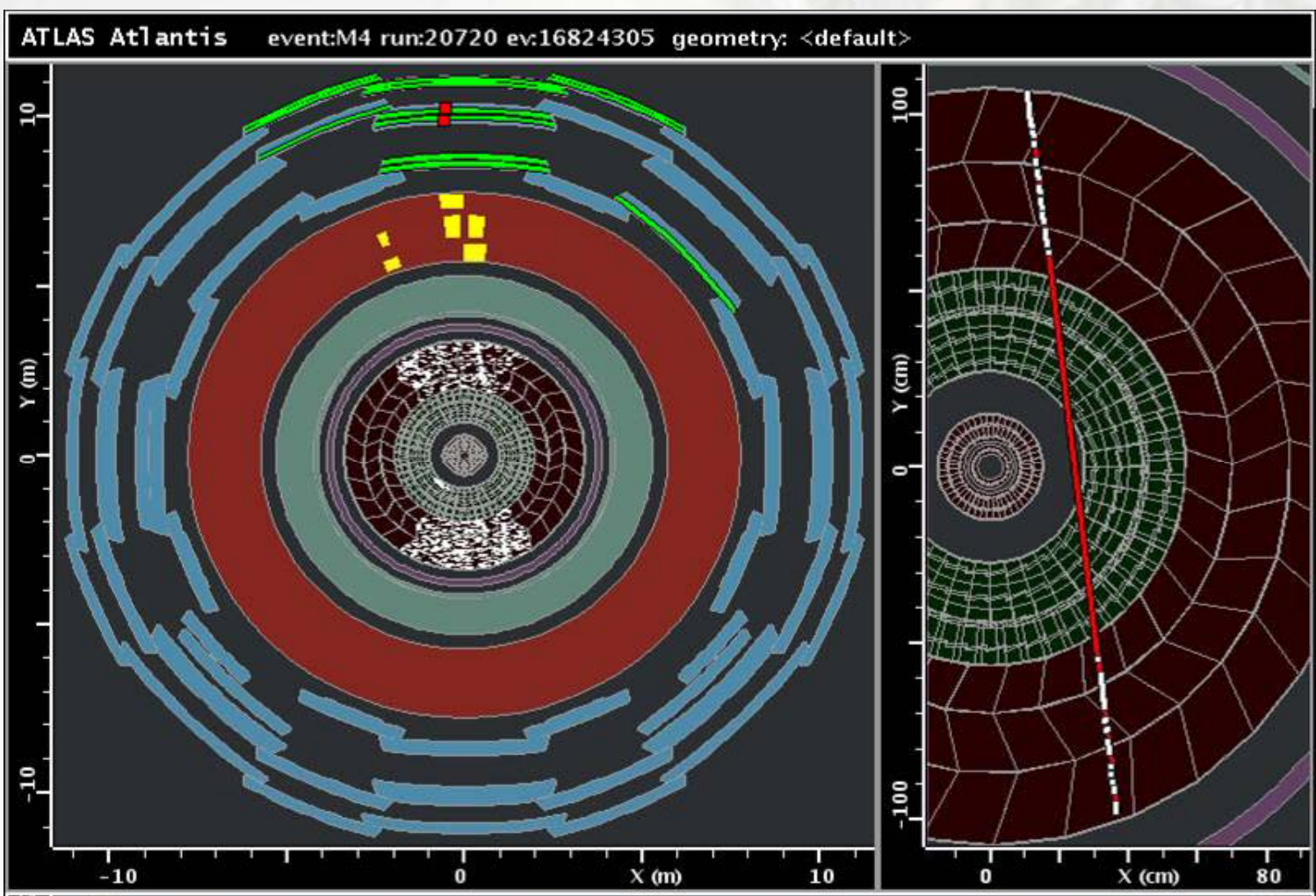

Example event from August run: cosmic through Muon system (MDT), Hadronic calorimeter (TileCal) and

ID Tracking (TRT)

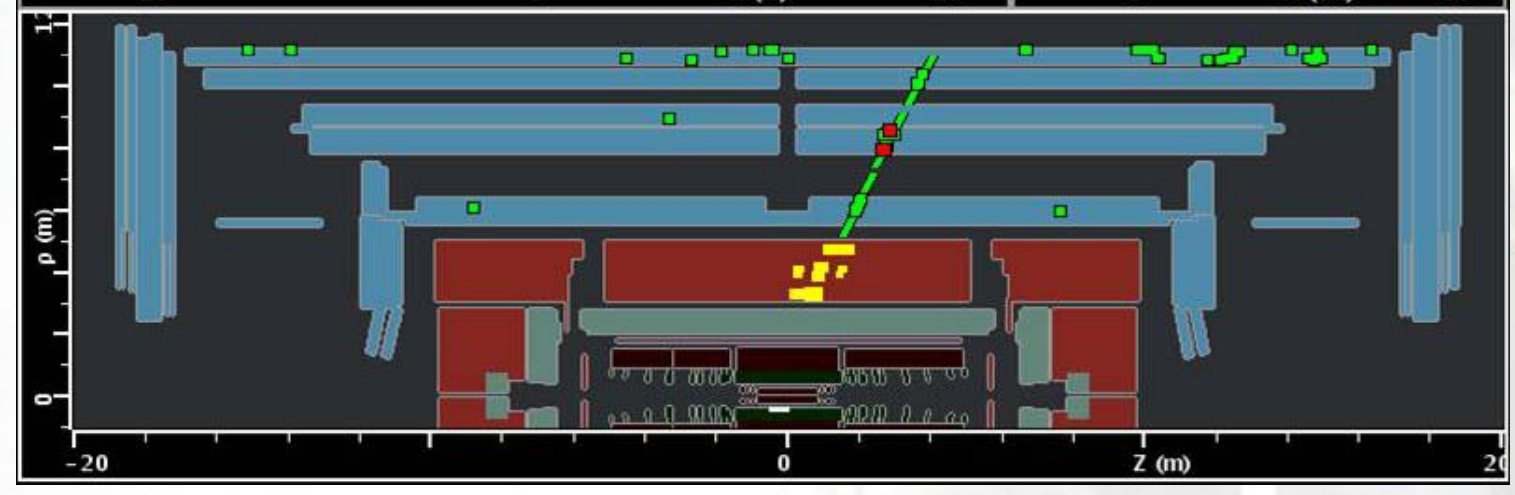

November run included:

- 1/4 of muon system

- $3 / 4$ of EMC

- 2/4 of HCAL 


\section{Global Commissioning Runs with Cosmics}

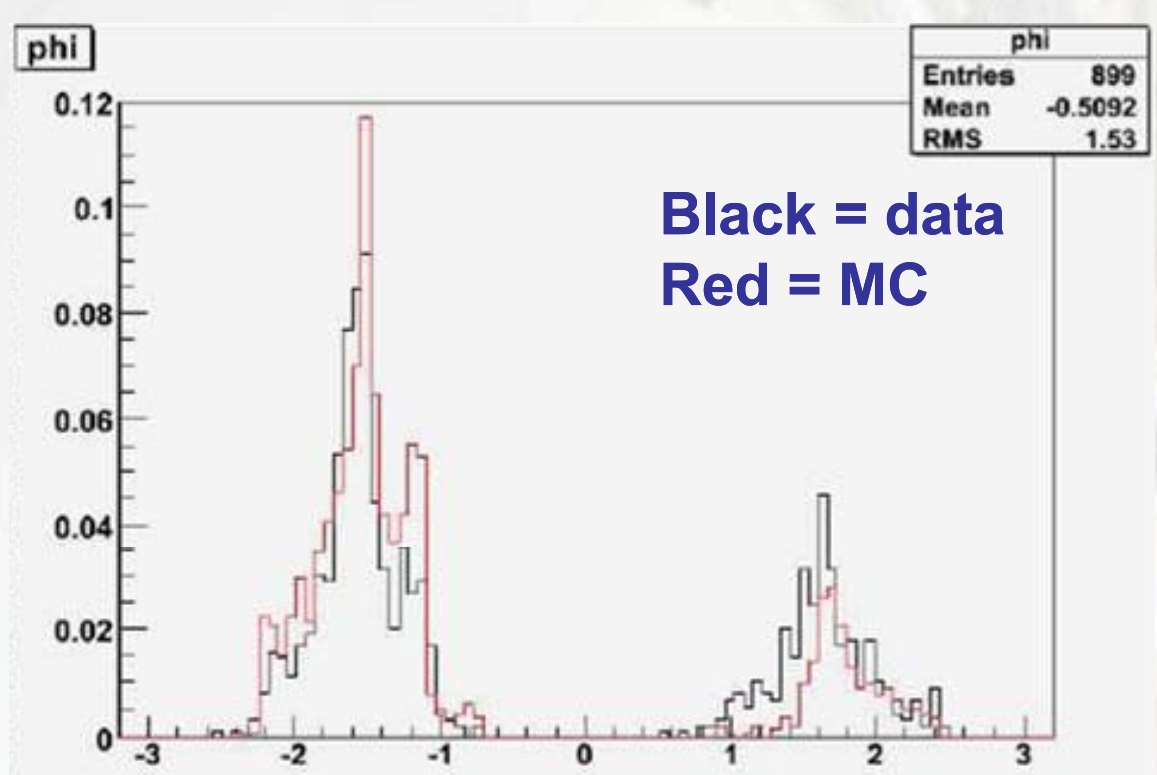

Phi-distribution of muons from TRT tracking, calorimeter and muon spectrometer combined, from November run:

- higher level trigger (software) operational

- monitoring of HLT operational

- detector performance understood

- Next run (March) will include SCT (inner detector silicon strip tracker) for first time ATLAS will migrate to a different mode of running two months before beam closure (=three months before first $14 \mathrm{TeV}$ collisions)

- will reach continuous (24/7) running when beam closed

- truly global cosmic runs

- commissioning with beam halo and beam gas interactions 


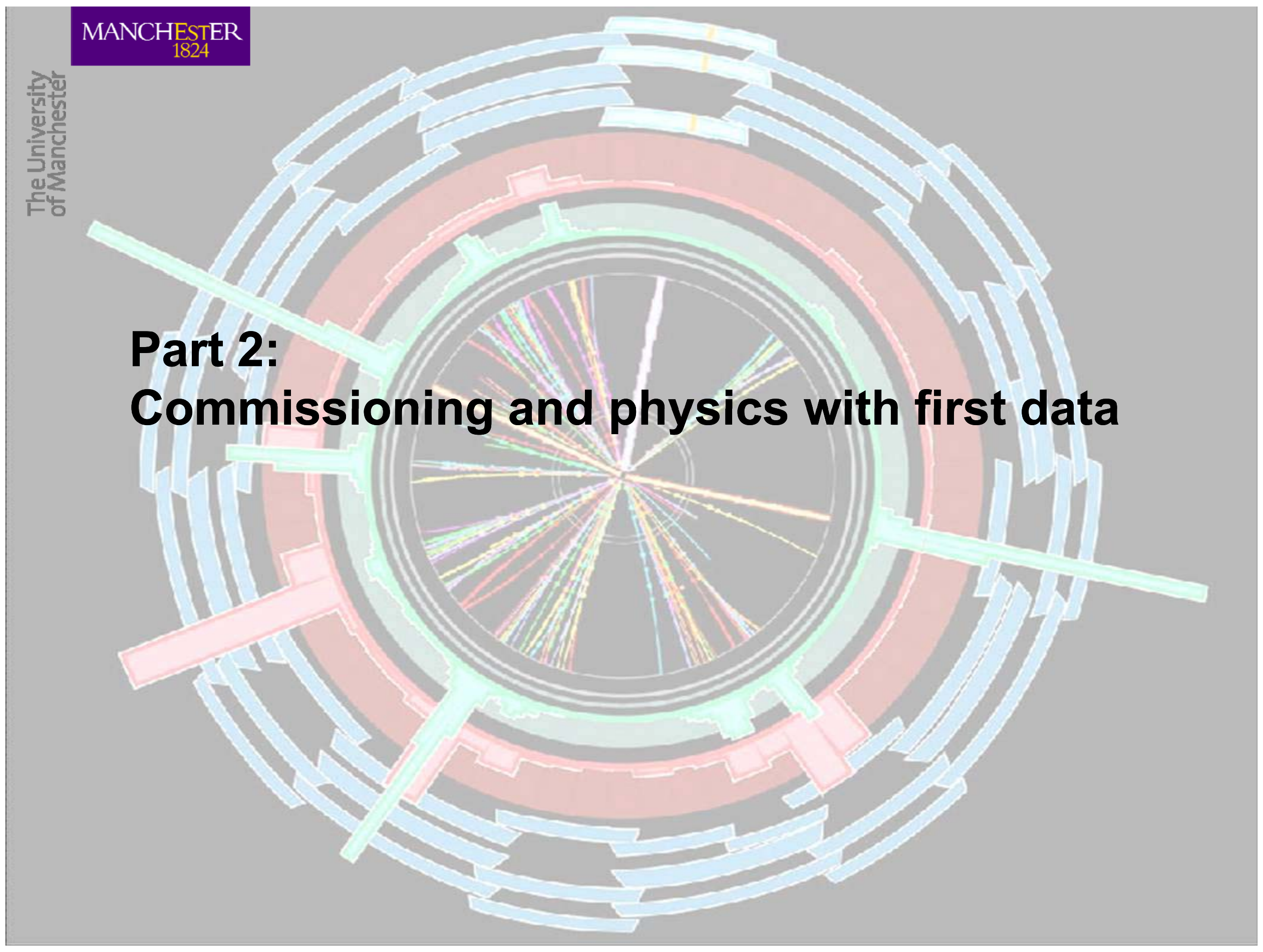




\section{The Road to Discovery}

Step \#1 - Understand / calibrate detector \& trigger in situ using well-known processes

- "Rediscover" Standard Model at $14 \mathrm{TeV}$

$\rightarrow$ use well modelled "standard candles": W, Z, top:

\begin{tabular}{|c|c|c|c|}
\hline Channel & $\begin{array}{l}\text { Events/ } \\
100 \mathrm{pb}^{-1}\end{array}$ & $\begin{array}{l}\text { Total Events from } \\
\text { LEP/Tevatron }\end{array}$ & Leads to understanding of... \\
\hline$Z \rightarrow e e, \mu \mu$ & $\sim 10^{4}$ & $\begin{array}{l}\sim 10^{6} \text { LEP } \\
\sim 10^{5} \text { Tevatron }\end{array}$ & $\begin{array}{l}\text { ECAL energy scale and uniformity } \\
\text { Tracking alignment }\end{array}$ \\
\hline $\mathrm{W} \rightarrow \mathrm{ev}, \mu \nu$ & $\sim 10^{5}$ & $\begin{array}{l}\sim 10^{4} \text { LEP } \\
\sim 10^{6} \text { Tevatron }\end{array}$ & $\begin{array}{l}\text { ECAL energy scale } \\
\text { Tracking alignment } \\
\text { Constrain PDFs }\end{array}$ \\
\hline $\mathrm{tt} \rightarrow \mathrm{WbWb} \rightarrow \mu \mathrm{v}+\mathrm{X}$ & $\sim 10^{2}$ & -104 Tevatron & $\begin{array}{l}\text { Jet scale from } W \rightarrow j j \\
B \text { tagging performance }\end{array}$ \\
\hline
\end{tabular}

Understand backgrounds to New Physics,

e.g. $t$ and $W / Z+$ jets (omnipresent...)

Step \#2

Look for New Physics potentially accessible in first year(s),

Step \#3 e.g. Z' $\rightarrow$ ee/ $\mu \mu$, SUSY, Higgs...? 


\section{Immediate New Territory...}

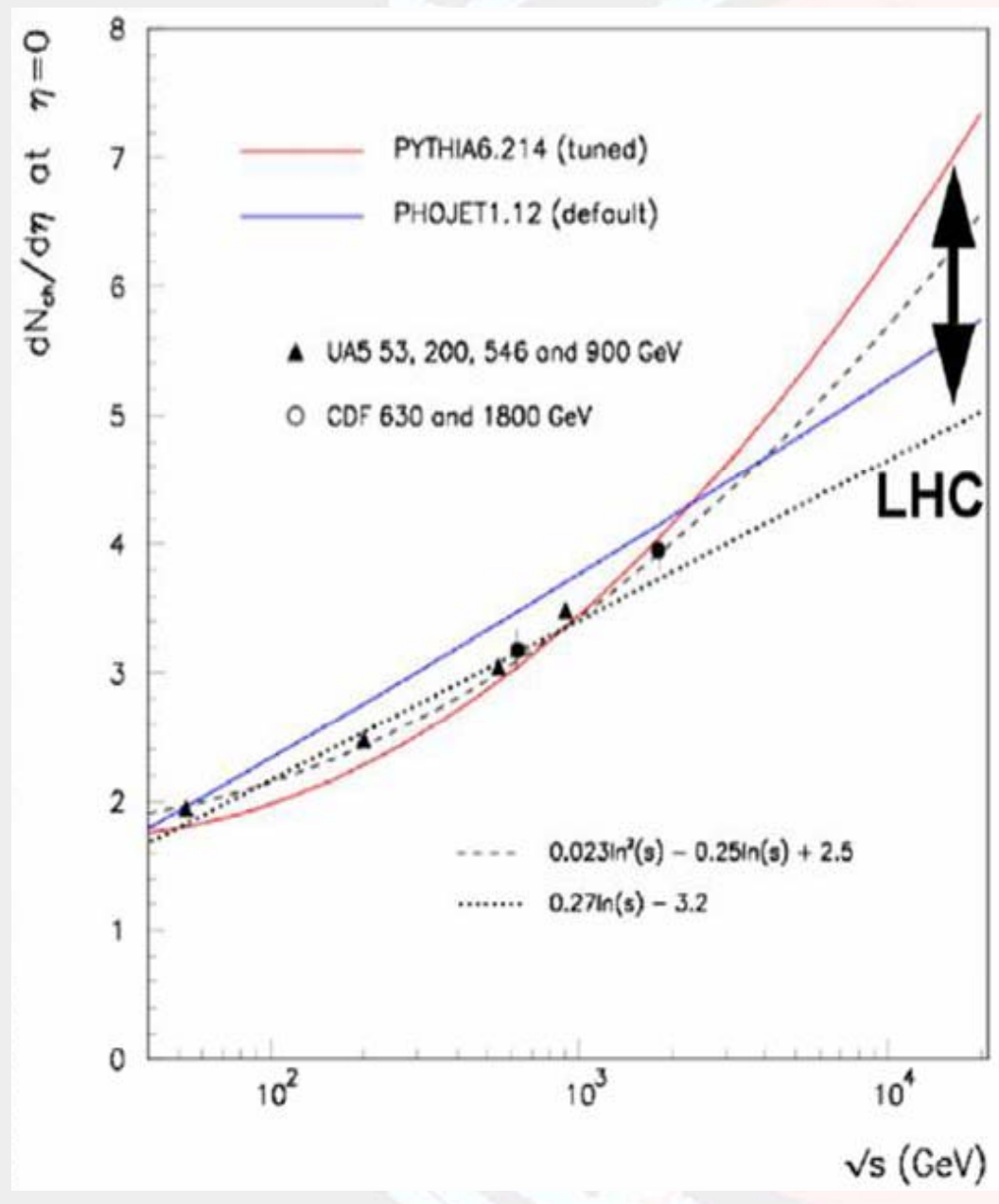

Minimum bias events: not well modelled at LHC energies

- Multiplicity of charged particles at $\eta=0$, as a function of centre-of-mass energy: extrapolating existing measurements to the LHC energies reveals differences of around $50 \%$ between the different models

- A sample of $\sim 10^{4}$ events represents sufficient statistics to measure the charged particle multiplicity with enough precision to discriminate between different models: in principle possible with a few hours' worth of data

- (Min. bias events will also play an important role in the trigger commissioning) 


\section{New Territory: QCD Jets}

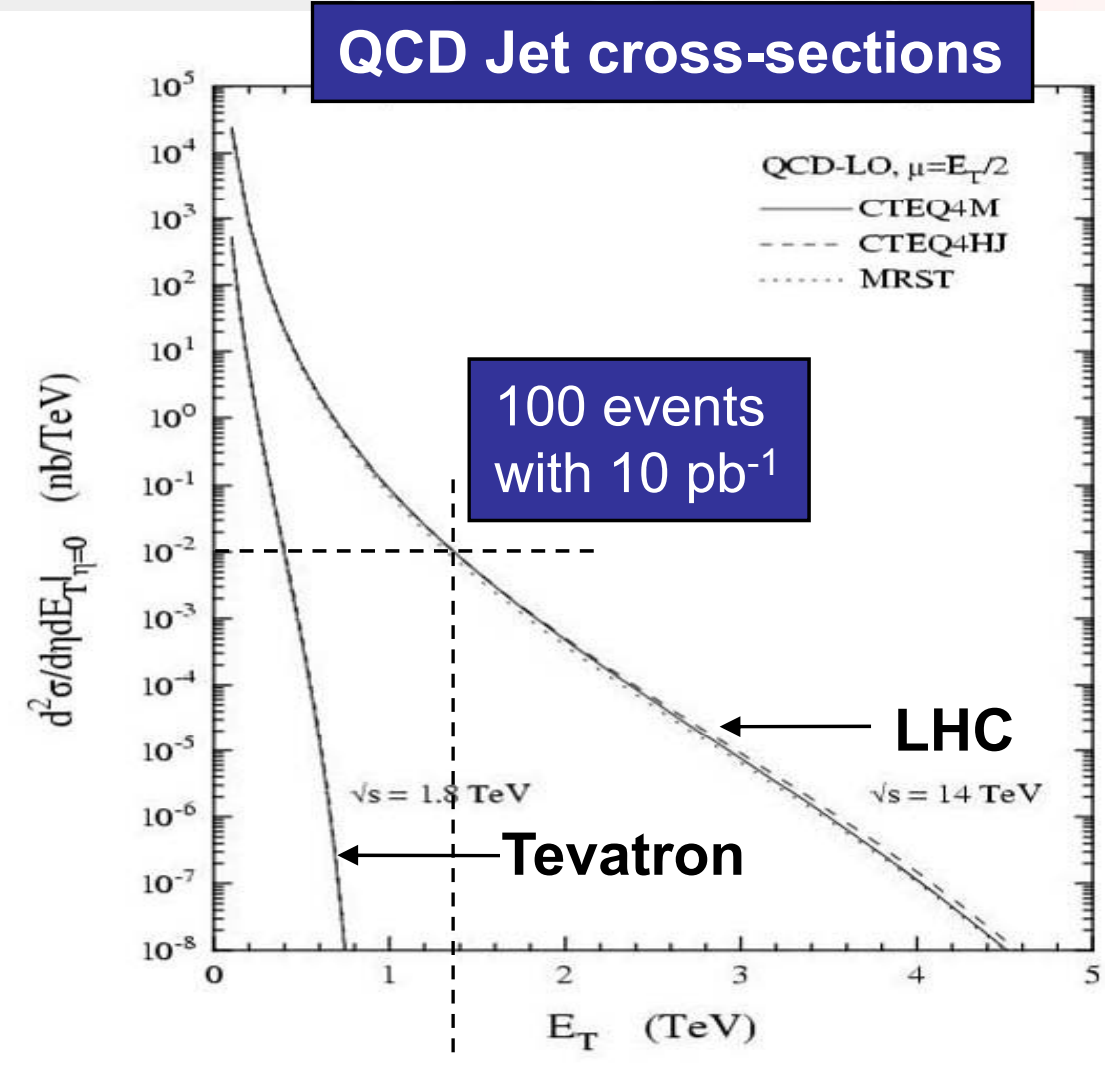

High cross-sections

( 100 events $/ 10 \mathrm{pb}^{-1}$ for $\left.\mathrm{E}_{\mathrm{T}}>1 \mathrm{TeV}\right)$

- rapidly probe QCD on a new scale

- high ET region sensitive to new physics

- background to all searches: accurate reconstruction and calibration is essential

Main error on jets cross-section is from jet energy scale

$-10 \%$ initially from test beam studies

$\rightarrow 1 \%$ from data $(\gamma / Z+j e t, W \rightarrow j j$ in $\mathrm{tt})$ 


\section{First Peaks and Detector Calibration}

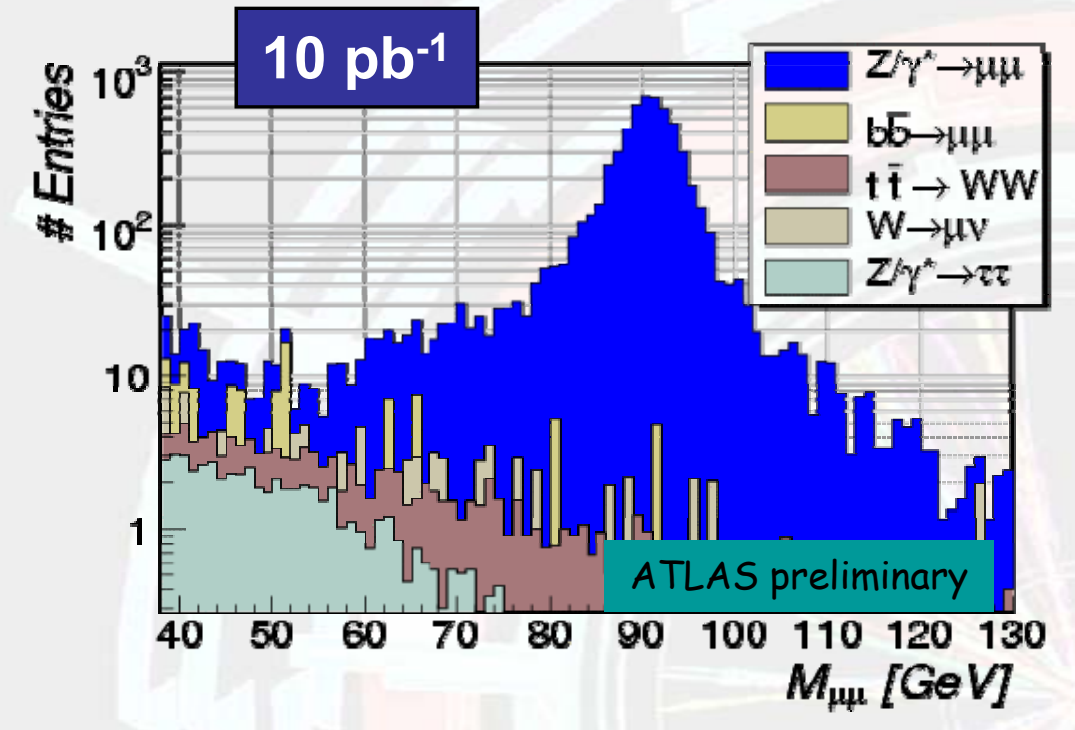

After all cuts expect $\sim 600 \mathrm{Z} \rightarrow \mu \mu /$ ee events per $\mathrm{pb}^{-1}$, per channel

Muon channel used for alignment of muon spectrometer and ID tracker, plus trigger and reconstruction efficiencies

Electron channel used to calibrate the lepton energy scale, using the $Z$ mass constraint, measure trigger and reconstruction efficiencies and calibrate

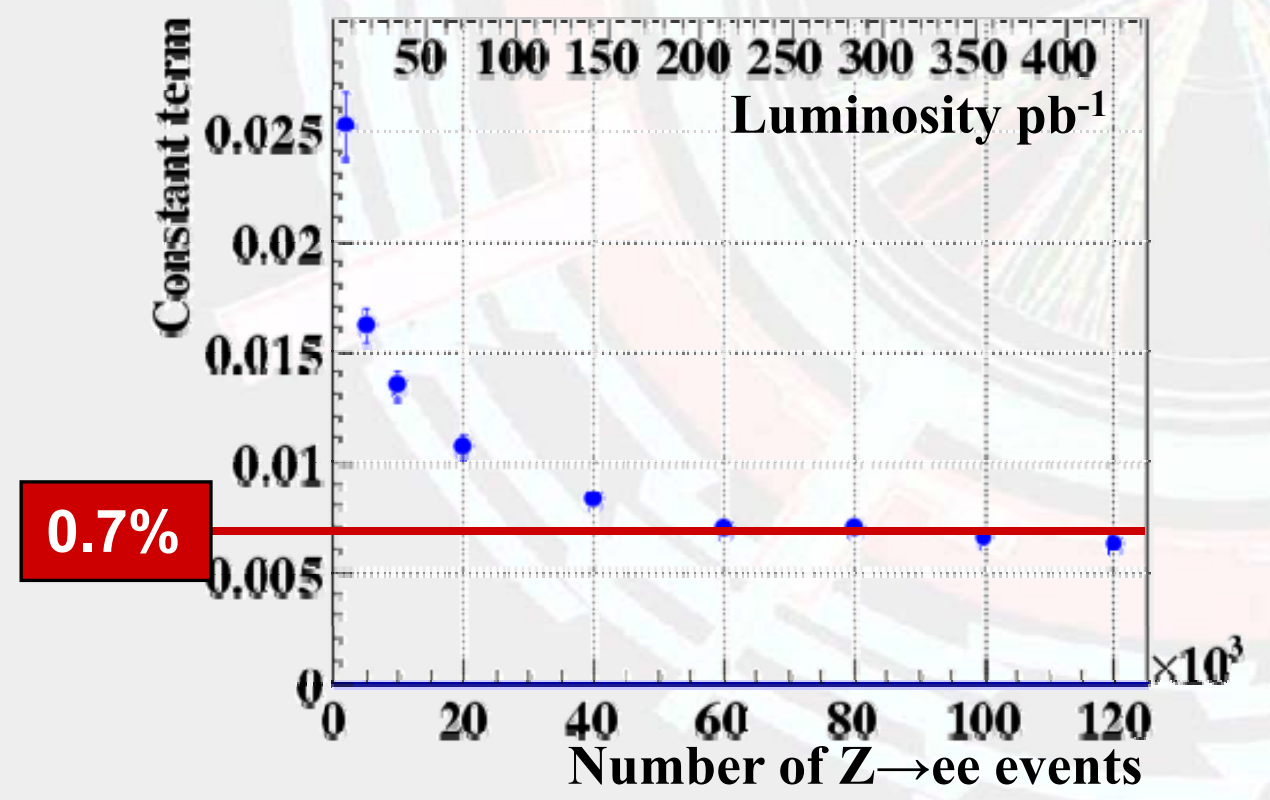
the ECAL uniformity:

- Expect initial, residual long range nonuniformities at level of $1-2 \%$

- Around $10^{5} Z \rightarrow$ ee events should be sufficient to reach the goal response uniformity of $\sim 0.7 \%$

With $100 \mathrm{pb}^{-1}$ the $Z$ cross section should be measured to around $10 \%$, dominated by the uncertainty on the luminosity 


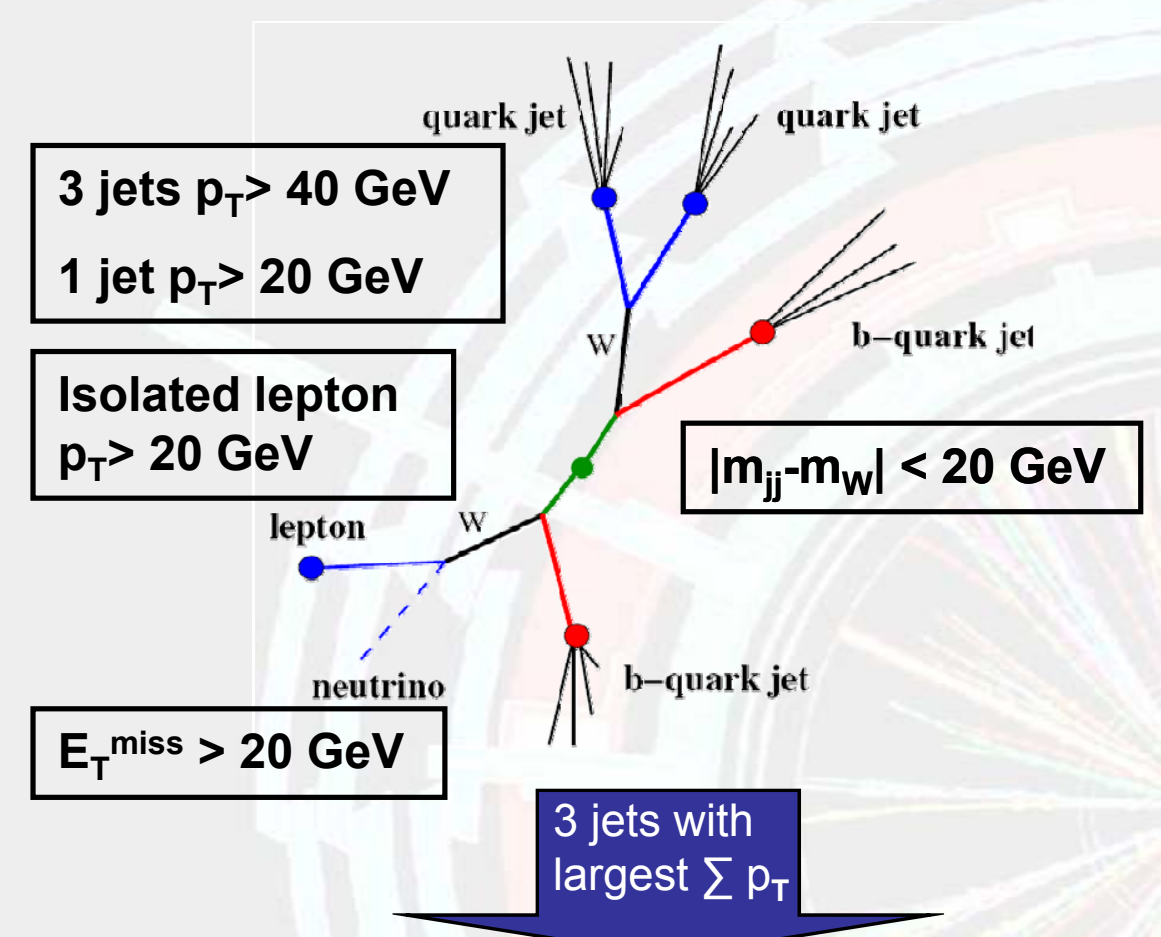

\section{Top Signal}

After all cuts expect $\sim 500$ events per $100 \mathrm{pb}^{-1}$ in the lepton plus jets channel ( $t \mathrm{t} \rightarrow$ blv bjj)

Signal can be observed with simple analysis without b-tagging

Error on cross-section is $\sim 20 \%$, dominated by luminosity and jet energy scale

NB: same events are used to calibrate the lightjet energy scale using the $W$ mass constraint $\left(M_{W}=M_{j j} K^{2}\right.$ where $K=$ jet energy scale factor $)$
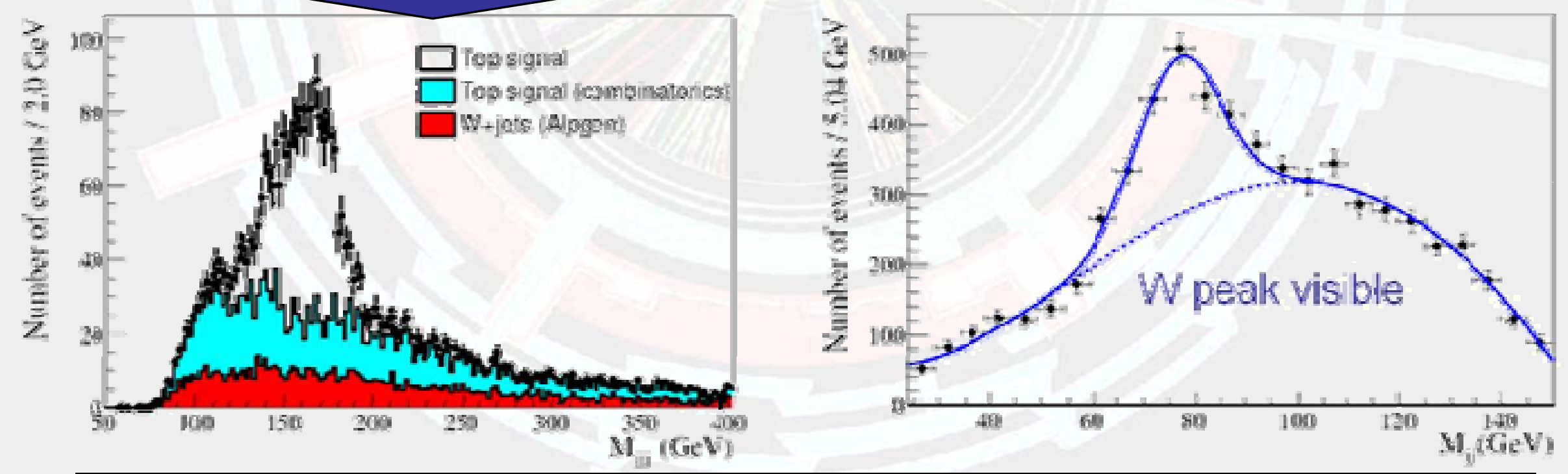


\section{Early Surprises?}

A very narrow Z' resonance decaying to $\mathrm{e}^{+} \mathrm{e}^{-}$is predicted in a "Higgsless" extension to the SM

For $\mathrm{M}_{\mathrm{Z}} \sim 1 \mathrm{TeV}$ this would be a very early discovery (ultimate reach is $5 \mathrm{TeV}$ with $300 \mathrm{fb}^{-1}$ ) (Could also be a graviton candidate but would need much more data to distinguish)

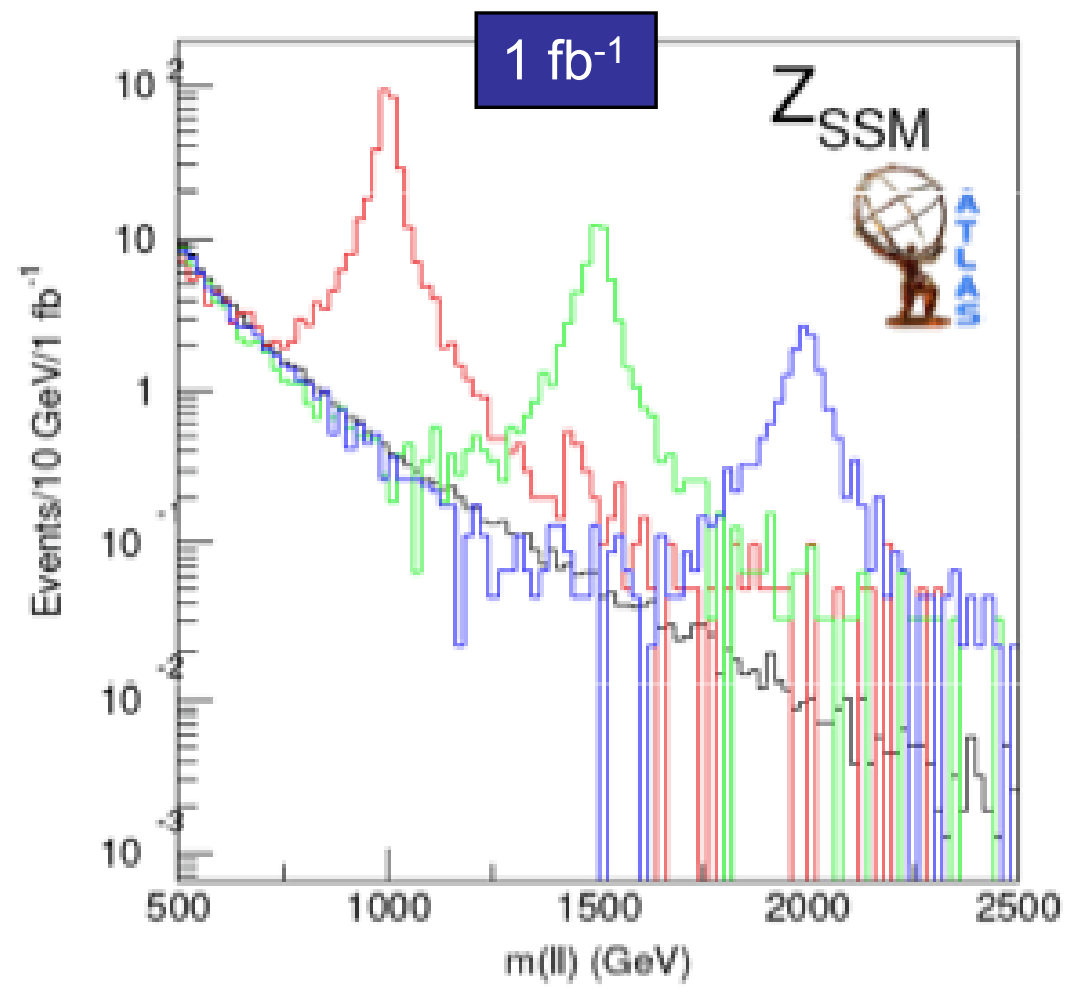

\begin{tabular}{|l|l|l|}
\hline Mass/TeV & $\begin{array}{l}\text { Selected } \\
\text { Events/fib-1 }\end{array}$ & JLfor Discovery \\
\hline 1 & $\sim 160$ & $\sim 70 \mathrm{pb}^{-1}$ \\
\hline 1.5 & $\sim 30$ & $\sim 300 \mathrm{pb}^{-1}$ \\
\hline 2 & $\sim 7$ & $\sim 1.5 \mathrm{fb}^{-1}$ \\
\hline
\end{tabular}




\section{Looking Ahead... SUSY?}

For squarks and gluinos with $\mathrm{M} \sim 1 \mathrm{TeV}$, hints of a signal can already show up with $100 \mathrm{pb}^{-1}$

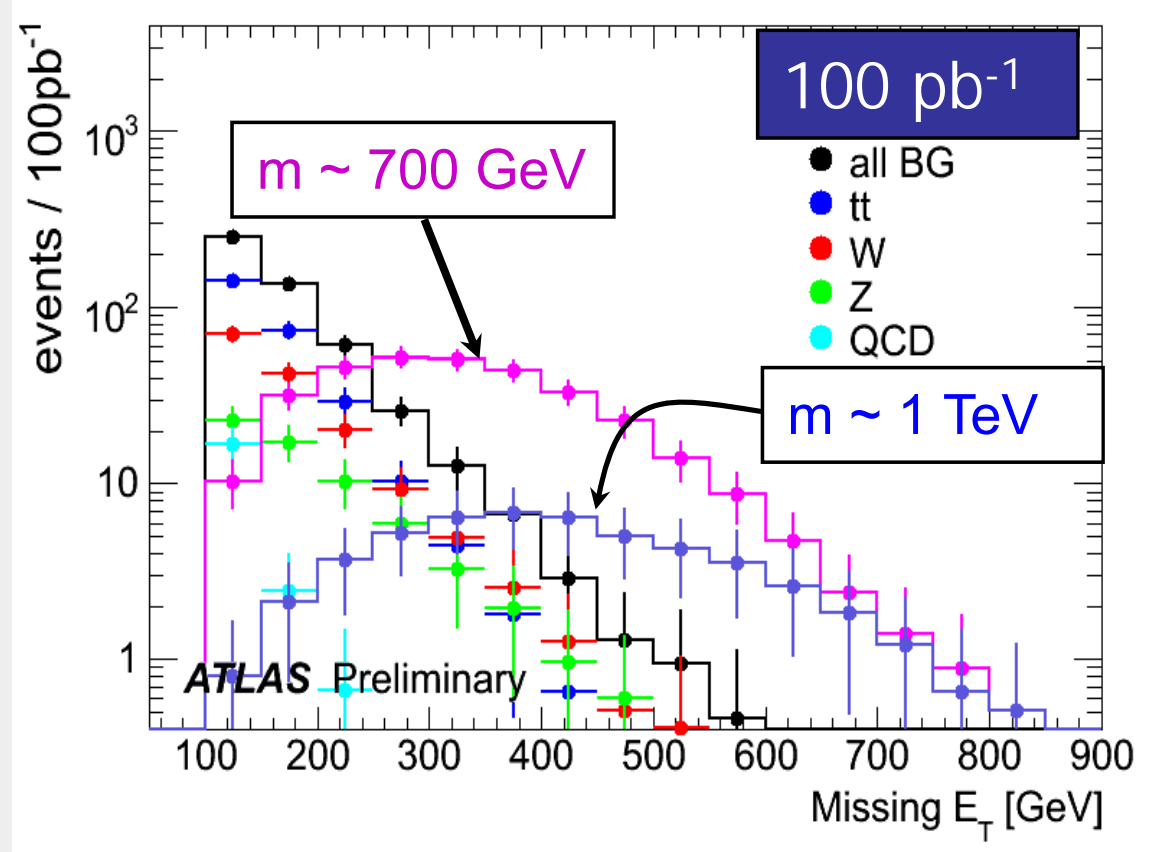

...but...

will need $\sim \mathrm{fb}^{-1}$ of data to understand the backgrounds which could fake this:

- $\mathrm{W} / \mathrm{Z}+$ jets with $\mathrm{Z} \rightarrow \mathrm{vv}, \mathrm{W} \rightarrow \tau \mathrm{v}$; $\mathrm{tt}$

- QCD multijet events with fake $E_{T}$ miss (calorimeter resolution, cracks, ...)

- cosmics, beam-halo, detector problems overlapped with high- $\mathrm{p}_{\mathrm{T}}$ triggers... 


\section{Finally, A Word on the Higgs...}

Higgs discovery will require $\sim \mathrm{fb}^{-1}$ of well understood data, with a light Higgs being the most difficult to observe: final word in 2010 ?

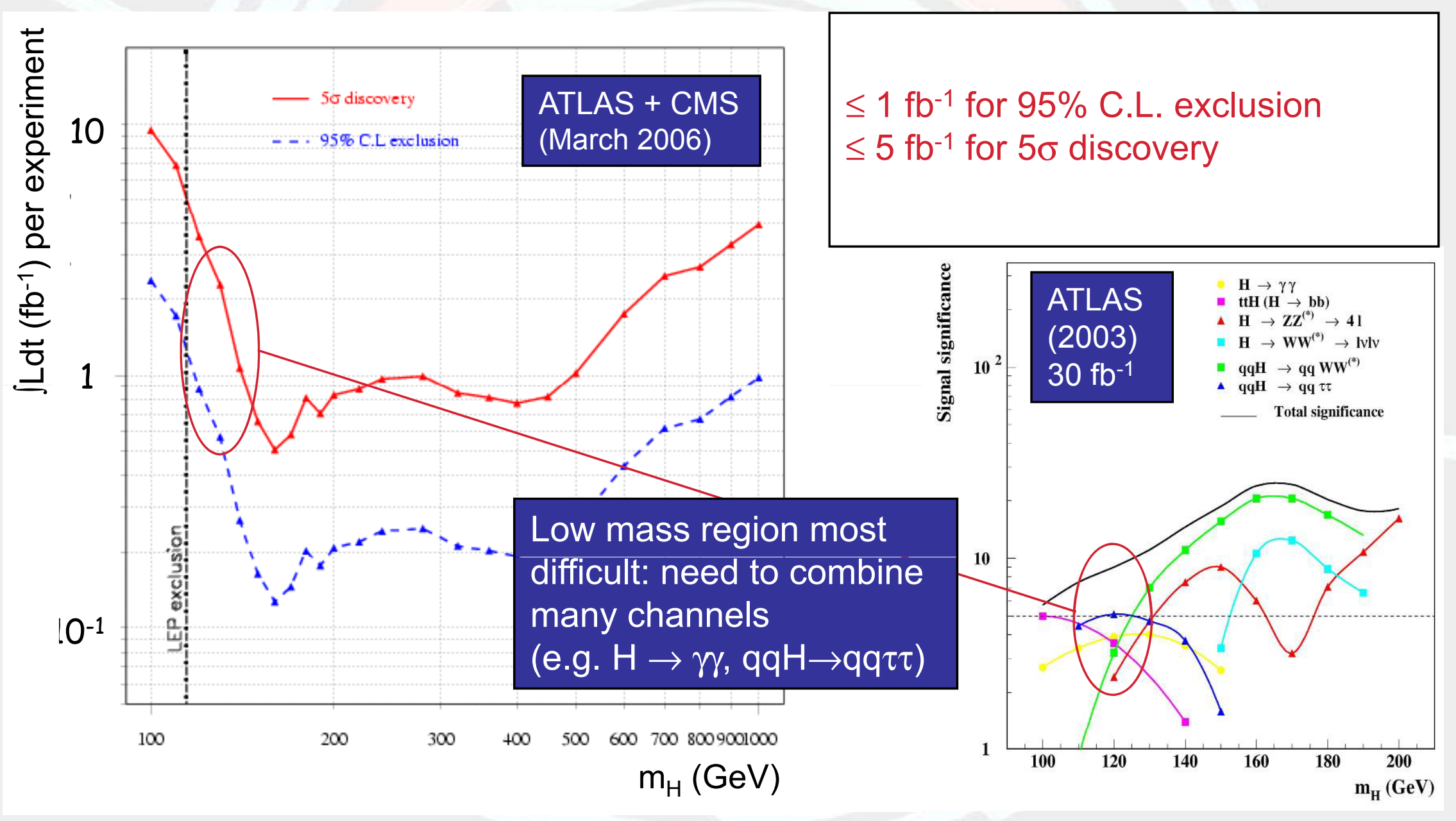




\section{Outlook}

\section{ATLAS readiness:}

- ATLAS is $~ 100 \%$ installed in the cavern

- Next few months will see remaining subsystems integrated into global TDAQ

- Will reach 24/7 operation with cosmics when beam pipe closed - ready and waiting for collisions!

\section{First Physics:}

- First 14TeV collisions during summer 2008

- Some immediate discoveries to be made on the nature of pp collisions at $14 \mathrm{TeV}$ - Well understood W, Z, top will be the first "rediscoveries" allowing the detector to be commissioned, calibrated and generally understood - hardware, trigger, offline reconstruction...

- The first steps on the road to discovery in 2008 